\title{
The Attitudes, Perspectives, and Barriers Among Primary Care Physicians Towards Addressing Sexual Health of Perimenopausal-Aged Women
}

\author{
Ali Farhat ${ }^{1}$, Laura Fulkerson, $\mathrm{DO}^{2}$, Brian Henriksen, $\mathrm{PhD}^{2}$ \\ Indiana University School of Medicine ${ }^{1}$; Fort Wayne Medical Education Program²
}

Background/Objective: Menopause is the natural cessation of menstruation that typically occurs in women between ages 45 and 50 . Menopause can lead to numerous issues regarding sexual health such as vulvovaginal atrophy, low desire, issues with vaginal lubrication, and an inability to achieve climax. Despite these issues, very few physicians initiate conversations about sexual health. Female patients generally feel uncomfortable bringing up the topic without first being asked by their physicians, so it is imperative to assess the reasons why physicians rarely discuss the topic.

Methods: Sixteen semi-structured interviews were conducted with family medicine physician faculty and residents regarding barriers to discussing sexual health with their patients. Interviews were transcribed, reviewed, and coded for common themes. Generalizable categories were identified from these themes.

Results: Patient embarrassment and cultural/religious norms were the most stated barriers to discussing sexual health. Adherence to norms and the lack of sexual education among patients were the most stated potential reasons for these barriers. The use of standardized questions and increasing efforts to directly discuss sexual dysfunction were the most stated potential solutions to these barriers. Better medical education regarding female sexual health and developing routines to ask about sexual health were the most stated strategies to help family medicine physicians to better serve this population.

Conclusion and Potential Impact: This study established, from a family medicine perspective, the barriers and respective potential solutions towards improving the sexual health of women of menopausal age. Patients with suboptimal sexual health can affect other aspects of their health. If barriers to discussing this topic with patients can be reduced, more sexual dysfunction diagnoses can be made, and family medicine physicians can better guide and treat patients to resolve these issues and improve overall quality of life. 